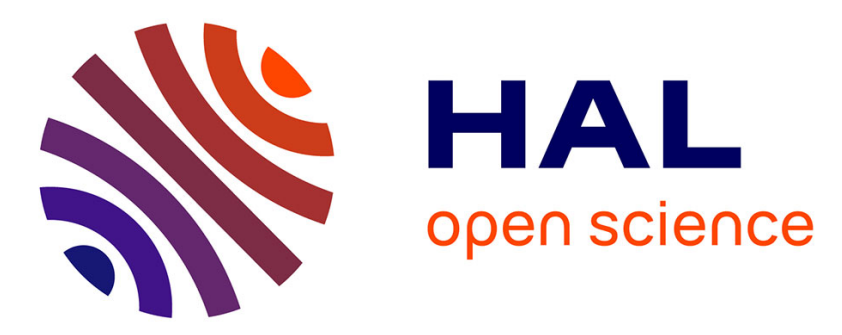

\title{
À la recherche d'un modèle éditorial: les revues de Giovanni Papini et les revues françaises à l'aube du XX e siècle
}

\author{
Alexia Kalantzis
}

\section{- To cite this version:}

Alexia Kalantzis. À la recherche d'un modèle éditorial: les revues de Giovanni Papini et les revues françaises à l'aube du XX e siècle. Revue de Litterature Comparee, 2014. hal-01894433

\section{HAL Id: hal-01894433 \\ https://hal.science/hal-01894433}

Submitted on 12 Oct 2018

HAL is a multi-disciplinary open access archive for the deposit and dissemination of scientific research documents, whether they are published or not. The documents may come from teaching and research institutions in France or abroad, or from public or private research centers.
L'archive ouverte pluridisciplinaire HAL, est destinée au dépôt et à la diffusion de documents scientifiques de niveau recherche, publiés ou non, émanant des établissements d'enseignement et de recherche français ou étrangers, des laboratoires publics ou privés. 


\section{À la recherche d'un modèle éditorial: les revues de Giovanni Papini et les revues françaises à l'aube du $\mathrm{XX}^{\mathrm{e}}$ siècle ${ }^{1}$}

$\mathrm{Au}$ début $\mathrm{du} \mathrm{XX}^{\mathrm{e}}$ siècle, la prolifération des périodiques favorise d'importants échanges entre la France et l'Italie. Le système d'abonnement et le rôle des bibliothèques assurent une bonne diffusion des périodiques à l'étranger. À cela s'ajoutent les comptes rendus des littératures réciproques et les contributions régulières d'écrivains étrangers dans les pages des revues. Ces transferts culturels sont soutenus par des correspondances entre les écrivains et par la présence importante des écrivains italiens à Paris. Les échanges sont particulièrement fertiles entre Paris et Florence. François Livi et Mario Richter ont souligné le rôle de la France pour de jeunes écrivains italiens comme Giovanni Papini et Ardengo Soffici, fondateurs de la revue Lacerba en $1913^{2}$, auxquels on pourrait rajouter Giuseppe Prezzolini, fondateur avec Papini de la revue Leonardo en 1903. Giovanni Papini et Ardengo Soffici ont tous deux séjourné à Paris et fréquenté les milieux des revues d'avant-garde. Soffici, durant son premier séjour, de 1900 à 1907, y accomplit sa «formation», selon le terme utilisé par Mario Richter dans le titre de son ouvrage ${ }^{3}$ : il se rapproche des milieux symbolistes, écrit pour La Plume et La Critique indépendante et fréquente Alfred Jarry, Félix Fénéon, Paul Fort, André Salmon, Guillaume Apollinaire et Pablo Picasso, tous assidus collaborateurs des périodiques parisiens. Parallèlement, Papini lit les revues françaises à Florence ${ }^{4}$ La ville toscane offre en effet de riches ressources en matière de littérature française, grâce au cabinet Vieussieux et à la bibliothèque ${ }^{5}$. L'importance des revues françaises pour les deux écrivains se reflètera dans leur propre revue, Leonardo, à travers la liste des périodiques reçus : en

\footnotetext{
${ }^{1}$ Cet article reprend une partie d'une communication proposée en décembre 2009 au séminaire TIGRE, consacré aux réseaux des revues et animé par Évanghelia Stead à l'ENS : «Le modèle de la petite revue entre France et Italie : autour du Mercure de France et de Leonardo». Sur les travaux du TIGRE, voir l'ouvrage dirigé par Hélène Védrine et Évanghelia Stead, L'Europe des revues (1880-1920), PUPS, 2008.

${ }^{2}$ Voir François Livi, «Tra Firenze e Parigi : il 'Leonardo' e la cultura francese », La Rassegna della letteratura italiana, série IX, ${ }^{\circ} 1$, janvier-juin 1905, p. 45-58. Voir également les différents articles sur le sujet regroupés dans Italica. L'Italie littéraire de Dante à Eugenio Corti, Lausanne, L'Age d'Homme, 2012. Sur les liens entre Soffici et la France, voir Mario Richter, La Formazione francese di Ardengo Soffici, Milan, Vita e Pensiero, 1969.

${ }^{3}$ Voir Mario Richter, La Formazione francese di Ardengo Soffici, ibid.

${ }^{4}$ Voir le journal de Papini, écrit en 1900, en partie en français, qui retrace ses lectures : Dario 1900, Florence, Vallecchi Editore, 1981.

${ }^{5}$ Fondé par Giovan Pietro Vieusseux en 1819, le « gabinetto scientifico-letterario » G.P. Vieusseux, plus qu'un cabinet de lecture, se veut un lieu de rassemblement pour les intellectuels italiens et étrangers. Sa bibliothèque s'enrichit au fil des années, avec de nombreuses éditions françaises et surtout des revues. Voir Gabinetto Scientifico G.P. Vieusseux, Prime edizioni francesi entrate in Biblioteca dal 1819 al 1918, présenté par Alessandro Bonsanti, Florence, Vallecchi, 1961, et Il Vieusseux : storia di un gabinetto di lettura 1819-2003: cronologia, saggi, testimonianze, dirigé par Laura Desideri, Florence, Polistampa, 2004.
} 
1905, la revue recense seize revues françaises dont le Mercure de France, Vers et Prose, La Plume, L'Ermitage, les Marges et La Revue des Idées ${ }^{6}$. Le Mercure de France en particulier constitue une référence importante en Italie, et plus largement en Europe. Selon François Livi, elle serait la plus lue des revues européennes ${ }^{7}$. Les chroniques des «Lettres étrangères », rédigées par des collaborateurs français ou étrangers, rendent compte des littératures du monde entier. Les comptes rendus sur la littérature italienne, d'abord rédigés ponctuellement par Remy de Gourmont au début des années 1890, se régularisent sous la plume de Luciano Zuccoli, puis sous celle de Ricciotto Canudo, et enfin de Giovanni Papini à partir de 1913. Ces échanges nourrissent le dialogue des idées et des formes. Comme l'a montré François Livi, Paris se présente comme un horizon culturel pour les jeunes écrivains florentins ${ }^{8}$. Or les revues parisiennes, au-delà de leur contenu, se présentent également comme des modèles éditoriaux. Dans cette perspective, nous souhaitons montrer plus particulièrement comme se tisse le réseau des revues entre Paris et Florence, en nous attardant d'abord sur l'expérience éditoriale de la revue moderniste, puis sur une figure privilégiée de passeur : Remy de Gourmont. Ce choix risque évidemment de fausser la perspective de l'étude du réseau des revues, en focalisant le point de vue sur une ville et sur un écrivain, mais il permet aussi de mettre en évidence une dynamique peu étudiée, et de souligner le lien entre les revues symbolistes et avant-gardistes.

\section{Les revues florentines du début du $\mathrm{XX}^{\mathrm{e}}$ siècle et le modèle éditorial de la « petite revue »}

\footnotetext{
${ }^{6}$ «Le Riviste che riceviamo », Leonardo, année III, n 3, juin-août 1905, p. 142.

${ }^{7}$ François Livi, « Tra Firenze e Parigi », art. cit., p. 56.

8 «Affermare che Parigi e la cultura francese rappresentano il principale punto di riferimento dei redattori del 'Leonardo' non significa cedere a una avventurata gallofilia, bensì rispettare un dato inoppugnabile : alla cultura d'otralpe la rivista fiorentina chiede la modernità e la legittimità. La modernità manifestata dalle grandi correnti di pensiero - filosofiche, letterarie e artistiche - che ermergono tra la fine Ottocento e il primo Novecento e che si impongono sulla ribalta europea; la legittimità di un consenso parigino, e quindi europeo, del quale il 'Leonardo', come ogni altra rivista italiana protonovecentesca, intende avvalarsi anche nei dibattiti nazionali. » [Affirmer que Paris et la culture française représentent le principal point de référence des rédacteurs du Leonardo ne signifie pas céder à une aventureuse gallophilie, mais respecter une donnée irréfutable : à la culture transalpine, la revue florentine demande la modernité et la légitimité. La modernité dont font preuve les grands courants de pensée - philosophiques, littéraires et artistiques - qui émergent entre la fin du XIX ${ }^{\mathrm{e}}$ siècle et le début du $\mathrm{XX}^{\mathrm{e}}$ siècle et qui s'imposent sur la scène européenne ; la légitimité d'un consensus parisien, et donc européen, dont le Leonardo, comme toute autre revue italienne du début du $\mathrm{XX}^{\mathrm{e}}$ siècle, entend aussi se servir dans les débats nationaux.]; François Livi, «Tra Firenze e Parigi », art. cit., p. 45-46. Nous traduisons toutes les citations. Anne-Rachel Hermetet a quant à elle montré l'inscription de la revue Leonardo dans une perspective européenne, à travers une conception particulière du romantisme. Voir «Romantisme et bergsonisme dans la revue florentine Leonardo (1903-1907), Romantisme, $\mathrm{n}^{\circ} 132$, « Rejet et renaissance du Romantisme à la fin du XIX ${ }^{\mathrm{e}}$ siècle », $2^{\mathrm{e}}$ trimestre 2006, p. 67-78.
} 
Leonardo : une revue moderniste dans la lignée des revues parisiennes fin-de-siècle

Giovanni Prezzolini et Giovanni Papini appartiennent à une génération que l'on pourrait qualifier de post-dannunzienne et qui revendique une place dans la littérature italienne. La création du Leonardo symbolise ainsi l'apparition dans le champ littéraire de nouveaux codes culturels inspirés de l'étranger. De nombreuses études ont souligné l'importance de Bergson pour Leonardo ${ }^{9}$. Mais des liens plus diffus existent entre la revue italienne et ses consoeurs françaises, qui ne sont pas moins importants pour la constitution de l'esthétique originale de Leonardo. Par ce dialogue avec les revues parisiennes, et plus largement avec l'Europe, la revue Leonardo tente de se détacher des modèles italiens pour s'engager dans une voie plus moderniste, qui passe par son contenu disciplinaire, mais aussi par son esthétique, sa dimension matérielle et sa rhétorique. La revue se positionne notamment par rapport à la revue romaine Il Convito et à la revue florentine Il Marzocco. Il Convito, fondé par Adolfo de Bosis en 1895, se présente sous la forme d'un recueil de littérature très loin du journal. D'Annunzio et Pascoli collaborent à cette revue-livre qui mêle des illustrations anciennes et modernes de Dante Gabriele Rossetti, G. Cellini, Andrea del Sarto, Francesco Paolo Michetti. Il Marzocco, «periodico settimanale di letteratura e d'arte», dirigé par Angiolo Orvieto, paraît à partir de 1896. Composé de quatre feuilles, avec des gravures, Il Marzocco est proche du journal par son format, mais rejoint néanmoins la revue par le travail sur la typographie et l'aspiration à la rareté bibliophilique. Ses positions antipositivistes le rapprochent du symbolisme européen, et sa grande référence reste D'Annunzio. Face à ces deux grands modèles que les jeunes auteurs considèrent comme trop académiques, Leonardo cherche une autre voie, à partir notamment des revues françaises, et plus précisément des « petites revues » auxquelles Papini fait d'ailleurs souvent référence en utilisant le terme français ${ }^{10}$. La revue paraît de façon irrégulière de 1903 (le premier numéro paraît le 4 janvier) à $1907^{11}$ et fonctionne sur le principe de l'adhésion. Elle débute à quinze adhérents, au tarif de 10 lires par mois. Le nom de la revue est inspiré par la figure de Léonard de Vinci, dont Papini et

\footnotetext{
${ }^{9}$ Voir par exemple A.R. Hermetet, art. cit. et Laura Schram Pighi, Bergson e il bergsonismo nella prima rivista di Papini e Prezzolini. Il «Leonardo». 1903-1907, Bologne, Arnaldo Forni, 1982.

${ }^{10}$ Voir par exemple l'article «Fiorentinità » dans Lacerba, 21 février 1915: «Firenze, sia bene o sia male, è la città delle riviste di giovanni, delle petites revues ed è questo uno dei caratteri che la fanno assomigliare a Parigi, anzi alla Rive gauche, a quella Rive gauche che i boulevardiers disprezzano o temono ma dalla quale sono venuti fuori i nomi più belli e più puri della letteratura francese da un secolo a questa parte. [Florence, que ce soit un bien ou un mal, est la ville des revues de jeunes, des petites revues, et c'est l'un des traits qui la font ressembler à Paris, et même à la Rive gauche, cette Rive gauche que les boulevardiers méprisent ou craignent, mais d'où sont sortis les noms les plus beaux et les plus purs de la littérature française depuis un siècle.] »

${ }^{11}$ La première série, qui passe de trois à un numéro par mois, s'interrompt en mai 1903. Une deuxième série débute le 10 novembre 1903, jusqu'en juin 1904, puis, après une nouvelle interruption, la revue connaît encore deux séries, de novembre 1904 à décembre 1905, puis de février 1906 à août 1907.
} 
Prezzolini retiennent la recherche de vérité, les écrits sur la vie et sur la beauté et l'esprit éclectique, comme le sera la revue. Le contenu est significatif de l'oscillation entre le journal et la revue, caractéristique des revues françaises de la fin du $\mathrm{XIX}^{\mathrm{e}}$ siècle $^{12}$. Les rubriques «Schermaglie» (escarmouches), polémique et ironique, «Alleati e Nemici » (alliés et ennemis), ou «Trucioli » (copeaux), à partir de la $2^{\mathrm{e}}$ série de la revue fin 1903, succèdent ainsi à des articles de fond et rappellent la parenté avec le journal par leur ton et leur forme. Il faut cependant noter l'absence de textes de création, puisque Leonardo se veut une « revue d'idées », avec un net accent philosophique, et non une revue «littéraire». Sur le plan matériel, la revue se différencie de ses prédécesseurs par un aspect «non moderne », inattuale selon Papini ${ }^{13}$. Leonardo est composé de huit grandes feuilles (in- $4^{\circ}$ ). Imprimée sur un élégant papier qui change de couleur au fil des livraisons (jaune, bleu, marron...), la revue est illustrée par des gravures sur bois et d'élégantes vignettes et cul-de-lampes mêlés au texte ${ }^{14}$. Le frontispice, de De Karolis, représente le soleil, emblème du rayonnement, et un aigle audessus d'une fontaine, avec l'inscription suivante : «Non si volge chi a stella è fissa ». À partir de 1906, pour des raisons financières, la revue évolue vers un format in- $8^{\circ}$, un papier plus commun et des illustrations plus rares ${ }^{15}$. Le frontispice change aussi de style, avec un dessin de Costetti, puis de Ghiglia, et enfin de Soffici ${ }^{16}$. Le style médiéval privilégié par la revue rappelle l'esthétique du Mercure de France, et, avant lui, de La Pléïade ${ }^{17}$, mais aussi de la revue L'Ymagier créée par Remy de Gourmont et Alfred Jarry en 1894, qui mêle l'ancien et le moderne avec des gravures médiévales et des œuvres de Gaughin, Filiger ou du Douanier Rousseau. La gravure sur bois, remise à l'honneur par ces écrivains français de la fin du siècle, trouve ainsi sa place dans les pages de la revue italienne. Ce caractère esthétique ancien contraste avec la revendication de la jeunesse, synonyme de modernité. Le programme

\footnotetext{
${ }^{12}$ Il faut rappeler que des revues comme le Mercure de France, La Plume ou La Revue blanche se caractérisent justement à l'époque par ce mélange entre la revue et le journal. Par exemple, la revue du mois, puis revue de la quinzaine dans le Mercure de France prend de plus en plus d'importance avec les années, inscrivant le Mercure dans l'actualité.

${ }^{13}$ Papini, Un uomo finito, [Florence, librairie de La Voce, 1913], Florence, Ponte Alle Grazie, 1994, p. 80-81.

${ }^{14}$ Selon Renato Bertacchini, les gravures sur bois étaient réalisées par les rédacteurs mêmes de la revue ( $L e$ riviste del Novecento. Introduzione e guida allo studio dei periodici italiani. Storia, ideologia e cultura, Florence, Le Monnier, 1979, p. 7).

${ }^{15}$ Voir Giorgio Luti, «Un editore fiorentino : Vallecchi», dans Firenze corpo 8, Vallecchi, 1983, p. 163 : «Il giornale dovette ridurre le pretese cambiando formato e uscendo ogni tre mesi in luogo di tre volte in un mese, ma sopratutto fu costretto a ricorrere alle più economiche fatture della Tipografia della Biblioteca di Cultura liberale [...] diretta dall'allora giovanissimo Attilio Vallecchi. » [Le journal dut réduire ses ambitions en changeant de format et en paraissant tous les trois mois au lieu de tous les mois, mais surtout, il fut contraint de recourir aux services de la plus économique Imprimerie de la Bibliothèque de Culture libérale dirigée par le très jeune Attilio Vallecchi]. Auparavant, Leonardo était imprimé par Giovanni Spinelli et Francesco Lumachi.

${ }^{16}$ Même après 1903, les couvertures de la revue sont très soignées, notamment avec une variation de couleurs, du marron au rouge, en passant par le vert.

${ }^{17}$ Notamment à travers les lettrines, les frises et les vignettes.
} 
de Leonardo développe en effet une rhétorique de la jeunesse typique des «jeunes revues » françaises, comme les dénommait Alfred Vallette ${ }^{18}$, et que l'on ne retrouve pas dans des revues italiennes comme Il Marzocco et Il Convito :

\begin{abstract}
Un gruppo di giovinni, desiderosi di liberazione, vogliosi d'universalità, anelanti a una superior vita intellettuale si son raccolti in Firenze sotto il simbolico nome augurale di Leonardo per intensificare la propria esistenza, elevare il proprio pensiero, esaltare la propria arte.
\end{abstract}

[Un groupe de jeunes, qui recherche la libération, qui aspire à l'universalité, qui désire ardemment une vie intellectuelle supérieure, se sont rassemblés à Florence sous le symbolique nom augural de Leonardo pour intensifier leur existence, élever leur pensée, exalter leur art. $]^{19}$

Cette déclaration liminaire, qui insiste sur la jeunesse comme une valeur en soi, est très proche des discours des jeunes revues françaises de la fin du XIX siècle, comme Le Scapin, La Pléiade, La Plume ou L'Art littéraire ${ }^{20}$. Dans Leonardo, cette thématique de la jeunesse est liée à une esthétique de la provocation. La revue se présente comme un périodique de combat, symbolisé par la vignette, récurrente, du chevalier qui brandit une lance. Elle deviendra par la suite spécifique à la rubrique critique des «Schermaglie» qui attaque des personnalités établies au moyen d'un langage provocateur. Cette dimension polémique s'inscrit plus généralement dans une culture collective fondée sur l'esprit ludique, comme le montre notamment l'utilisation des pseudonymes: Gian Falco (Papini), Giuliano il Sofista (Prezzolini) ${ }^{21}$, Augustus (Augusto Mussolini), de Karolis (De Carolis), Perseo (Giovanni

\footnotetext{
${ }^{18}$ Voir la rubrique tenue par Vallette dans L'Écho de Paris littéraire et illustré d'octobre 1892 à août 1893. Sur les questions d'appellation et la critique de l'étiquette «petite revue», voir Évanghelia Stead, « Les revues avant la NRF : raisons d'une science difficile», communication au colloque international La Nouvelle Revue Française, naissance d'un mythe, Bourges, 18-20 juin 2009, dans La Nouvelle Revue française. Les colloques du centenaire, dir. A.-R. Hermetet et W. Marx, Gallimard, coll. « Cahiers de la NRF », 2013, p. 105-117.

${ }_{19}$ «Programma sintetico », Leonardo, année I, ${ }^{\circ} 1,4$ janvier 1903, p. 1.

${ }^{20}$ Voir par exemple le «colloque préliminaire » de L'Art littéraire $\left(\mathrm{n}^{\circ} 1\right.$, octobre 1892$)$ : «Que pouvez-vous représenter ? Les jeunes, qui ne le sont pas encore : je parle des littérateurs... Tous ces messieurs de trente ans qui encombrent les rédactions ne sont plus des jeunes, mais des ratés [...] Il y aura désormais, en dehors de toute école, un recueil ouvert aux Vrais Jeunes, aux tout derniers venus qui montreront du talent... » $\mathrm{Ce}$ programme, rédigé par Louis Lormel sous forme de dialogue ludique, rejoint ces quelques lignes de Papini à propos de Leonardo : «Il tante volte proposto e disegnato giornale che deve raccogliere le impazienze degli ignoti, dar voce e figura a un manipolo d'oscuri, rivelare ai maestri immediati ; ai non più giovani, agli uomini di trenta e quarant' anni che i veri giovanni, i freschi giovani di vent'anni, son arrivati anche loro alla maggior età e che un'altra generazione ha finalmente diritto alla parola. » [Le journal tant de fois proposé et projeté, qui doit recueillir les impatiences des inconnus, donner voix et figure à une poignée d'obscurs, révéler aux maîtres immédiats, à ceux qui ne sont plus jeunes, aux hommes de trente et quarante ans, que les vrais jeunes, les frais jeunes de vingt ans sont eux aussi arrivés à l'âge majeur et qu'une autre génération a finalement le droit à la parole.] (Un uomo finito, éd. cit., p. 73).

${ }_{21}$ Avant même la création de la revue, Papini et Prezzolini utilisent déjà ces pseudonymes dans leur correspondance. Voir Giovanni Papini-Giuseppe Prezzolini, Carteggio. I, 1900-1907, a cura di Sandro Gentili, Gloria Manghetti, Rome, Edizioni di storia e letteratura ; Lugano, Biblioteca Cantonale, Archivio Prezzolini, 2003.
} 
Costetti), Pholos Centauro (Mario Venturini), Belfegor (Felice Marchionni), La Compiuta Donzella (Amy Bernardy), Ortensio (Emilio Cecchi), Piero Eremita (Piero Marucchi). Ce dernier exemple rappelle évidemment les pseudonymes utilisés dans L'Ermitage. Plus généralement, la mode du pseudonyme se retrouvait dans les jeunes revues comme le Mercure de France, avec le collectif Quasi, les multiples pseudonymes de Remy de Gourmont (Lucile Dubois, R. de Bury...), ou celui de Paul Léautaud (Maurice Boissard), souvent prétextes à des clins d'œil ludiques pour le lecteur averti. Dans la revue italienne, le jeu sur les pseudonymes s'accompagne d'une utilisation graphique, les signatures prenant la forme de vignettes propres à chaque collaborateur, comme le faucon pour Gian Falco, le cheval pour Giuliano il Sofista, ou la fontaine pour de Karolis. Ce procédé avait été esquissé dans le Mercure de France des années 1890, avec des vignettes qui parfois revenaient pour un auteur. Le Mercure de France apparaît d'ailleurs comme une référence dans le domaine éditorial, avec sa maison d'édition. La «bibliothèque du Leonardo », l'une des premières entreprises éditoriales autonomes liées à une revue à Florence, s'inspire de celle de la revue française :

\begin{abstract}
Già da qualche tempo ci siam persuasi che il solo Leonardo non basta per dire tutto quello che vogliamo dire e per esercitare tutta quella influenza che siamo costretti ormai ad esercitare. E pensammo, or sono due anni, a creare, accanto al Leonardo, una collezione di apuscoli, di pamphlets, di libri scritti da noi stessi e dai nostri amici (sia italiani che stranieri). Volevamo fare, cioè qualcosa di simile alle editions du Mercure de France o a quelle di Diederich vale a dire una collezione piuttosto ristretta ma dove potessero stare insieme, senza troppo vergognarsi l'un dell'altro, libri di filosofia e di poesia, di novelle e di teorie scientifiche.

[Déjà depuis quelque temps nous sommes convaincus que le seul Leonardo ne suffit pas pour dire tout ce que nous voulons dire et pour exercer toute l'influence que nous sommes désormais obligés d'exercer. Et nous pensons, après deux années, à créer, à côté du Leonardo, une collection d'opuscules, de pamphlets, de livres écrits par nous-mêmes et par nos amis (qu'ils soient italiens ou étrangers). Nous voulions donc faire quelque chose de semblable aux éditions du Mercure de France ou à celles de Diederich, c'est-à-dire une collection plutôt réduite mais où puissent cohabiter, sans trop avoir honte l'un de l'autre, des livres de philosophie et de poésie, de nouvelles et de théories scientifiques. $]^{22}$
\end{abstract}

Papini et Prezzolini sont donc particulièrement attirés par la dimension encyclopédique de la revue française, qui s'adapte bien à leur projet de « revue des idées $»^{23}$. Leonardo se veut ouvert aux différents domaines de la connaissance, tout comme en France la contemporaine Revue des idées.

\footnotetext{
${ }^{22}$ Leonardo, année III, $\mathrm{n}^{\circ} 3$, octobre-décembre 1905.

${ }^{23}$ Sur l'entreprise éditoriale de Leonardo, voir Giorgio Luti, « Un editore fiorentino : Vallecchi », art. cit., p. 161 et suivantes, et Luca Brogioni, « 'La Voce', la rivista che volle farsi editore », La fabbrica del libro, bollettino di storia dell'editoria in Italia, année XIV 2/2008, p. 26-27 (article mis en ligne sur le site www.fondazionemondadori.it, consulté le 5/02/13).
} 
La Revue des idées, dirigée par Remy de Gourmont et Édouard Dujardin, paraît à partir de janvier 1904. Elle apparaît comme une revue satellite du Mercure de France : outre la figure de Gourmont qui occupe une place importante dans le Mercure, elle est dirigée administrativement par Alfred Vallette, directeur du Mercure. Son titre fait écho au sous-titre de Leonardo, « rivista d'idee », adopté à partir du 10 novembre 1903, ce qui n'est sans doute pas un hasard : Ricciotto Canudo consacre quelques comptes rendus à la revue italienne, bien connue de Gourmont, dans les pages du Mercure ${ }^{24}$. Le principe de ces deux revues est similaire. Il s'agit de créer une revue proche de la revue littéraire, mais non littéraire. Leonardo apparaît ainsi comme une revue qui oscille entre la littérature et la philosophie, et La Revue des idées entre la littérature et la science. Les deux revues prônent ainsi le décloisonnement des disciplines, contre l'académisme. Elles se rejoignent également dans une forme d'encyclopédisme. Il est donc tout naturel que Leonardo salue dès sa parution $L a$ Revue des idées :

\begin{abstract}
La Revue des idées ci sembra un opera di Voltairianismo, antiscientifico, diretta da quello spirito spesso profondo e quasi sempre acuto di Rémy de Gourmont, con la scusa di far conoscere ai letterari il dominio della scienza, è in realtà la raccolta di tutte le idee e di tutti i fatti che possono disturbare, pungere, urtare i quieti e creduli scienzati. [...] Raccolta di malignità anti-scientifiche, fatte con le stesse armi della scienza, la Revue des idées pel suo titolo, per la sua forma, pel suo fine non potrebbe esserci più simpatica.

[La Revue des idées nous semble une œuvre de voltairisme, antiscientifique, dirigée par cet esprit souvent profond et toujours perspicace qu'est Remy de Gourmont, avec l'excuse de faire connaître aux littéraires le domaine de la science, il s'agit en réalité de réunir toutes les idées et faits qui peuvent déranger, titiller, heurter les tranquilles et crédules hommes de science. [...] Recueil de malignité anti-scientifique, fait avec les armes mêmes de la science, la Revue des idées, pour son titre, pour sa forme, pour ses objectifs, ne pourrait nous être plus sympathique. $]^{25}$
\end{abstract}

Par la suite, une plus grande place est accordée à la science dans Leonardo, à travers notamment la contribution de Giovanni Vailati, philosophe mathématicien. La revue mentionne souvent La Revue des idées et se montre particulièrement attentive aux parutions des collaborateurs de la revue de Gourmont. Par exemple, en juin 1904, Papini rend compte de l'ouvrage de Georges Palante, Combats pour l'individu (Alcan, 1904). Dans le numéro suivant, en novembre, la revue publie la réponse de Palante à travers une lettre, suivie par la réponse de Papini, tout cela étant regroupé sous le titre «Contrasti individuali ». Palante devient presque un collaborateur de la revue. Cela n'est pas sans rappeler la stratégie publicitaire de Poesia: il s'agit de montrer l'influence de la revue et son rayonnement,

\footnotetext{
${ }^{24}$ Voir par exemple le Mercure de France du $1^{\text {er }}$ février 1905, p. 474.

${ }_{25}$ «La mitologia della scienza», compte rendu de Giuliano il Sofista dans la rubrique «Alleati e nemici», Leonardo, année II, $\mathrm{n}^{\circ} 1$, mars 1904 , p. 26. Ce compte rendu est d'ailleurs suivi d'une note du même auteur sur «Lo spiritualismo scientifico », qui rattache les préoccupations de la revue de Gourmont à celles de Leonardo.
} 
notamment en France, en affichant les noms de tout écrivain qui mentionne d'une façon ou d'une autre la revue ${ }^{26}$. Il faut également souligner le fait que la lettre de Palante est publiée en français, ce qui pose la question du plurilinguisme. Le français est régulièrement utilisé dans Leonardo, qui vise ainsi non seulement un public français, mais aussi une visibilité internationale ${ }^{27}$.

\title{
Le dépassement du modèle éditorial de la «petite revue »
}

Il faut cependant insister sur l'ambiguité qui fonde dès le départ le projet de Papini et Prezzolini : la revue Leonardo naît d'une volonté de renouvellement des lettres italiennes qui passe certes par une inscription dans le domaine européen, mais qui vise à revaloriser l'Italie et à garantir son indépendance culturelle. On peut ainsi lire l'évolution de Leonardo à Lacerba comme l'affirmation progressive d'un nationalisme culturel et comme l'invention d'un modèle italien, voire florentin, de la revue. Dès 1907, Giovanni Papini mesure les limites d'une revue comme Leonardo, dont il choisit délibérément d'arrêter la publication. Il évoque d'abord, paradoxalement, le trop grand succès de la revue, et donc le refus du caractère commercial qui lui est indissociable :

\begin{abstract}
Il Leonardo non ha mai avuto, nella nostra mente, niente di commerciale e l'idea di avere accanto degli amministratori, e magari degli azionisti, i quali più o meno direttamente ci avvrebbero forzato a modificare lentamente la nostra impresa per accrescere o per non diminuire gli utili, ci repugna completamente. Noi abbiamo scritto sempre per pochi, sapendo bene che le cose da noi dette potevano essere comprese e vissute solo soltanto da chi avevano anime ed esperienze simili alle nostre, e questa frotta di abbonati professori, dottori, avvocati, dilettanti che andava crescendo intorno a noi ha finito con lo annoiarci. Anche involontariamente un giornale diventa ciò che vogliono i suoi lettori e per quanto abbiamo resistito abbastanza fino ad oggi sarebbe impossibile continuare a far concessioni a quelli che ci seguono.
\end{abstract}

[Leonardo n'a jamais rien eu, dans notre esprit, de commercial, et l'idée d'avoir à nos côtés des administrateurs, et peut-être même des actionnaires, qui nous auraient plus ou moins directement forcés à modifier lentement notre entreprise pour accroître ou diminuer les bénéfices, nous répugne tout à fait. Nous avons toujours écrit pour un petit nombre, en étant parfaitement conscients que les choses que nous disions pouvaient être comprises et vécues seulement par ceux qui avaient des âmes et des expériences similaires aux nôtres, et cette bande de professeurs, docteurs, avocats, dilettantes abonnés qui augmentait autour de nous a fini par nous ennuyer. Même involontairement, un journal devient ce que veulent ses

\footnotetext{
${ }^{26}$ Dans Poesia, la stratégie est poussée à l'extrême : tout auteur d'une lettre, ou d'un commentaire parfois très critique sur la revue, devient sous la plume de Marinetti un collaborateur de la revue. Voir les analyses de François Livi dans « Marinetti et 'Poesia' (1905-1909). Du liberty au futurisme», dans L'Italie littéraire, op. cit., p. 307-308.

${ }^{27}$ On peut citer par exemple l'article de Pierre Roché sur l'Ermitage, en février 1905, qui développe la notion d' « européen » au sujet de ses collaborateurs : «Plusieurs des noms qui précèdent ne sont-ils pas déjà Européens, au sens qui nous est cher ?» (Leonardo, année III, ${ }^{\circ} 1$, février 1905, p. 30).
} 
lecteurs et même si nous avons résisté assez bien jusqu'à aujourd'hui, il serait impossible de continuer à faire des concessions à ceux qui nous suivent. $]^{28}$

On pense ici au succès du Mercure de France, qui passe de cinquante abonnés environ en 1890 à 3000 lecteurs en 1905 et qui atteint une forme de reconnaissance sociale avec des lecteurs prestigieux et une importante diffusion à l'étranger ${ }^{29}$. Alfred Vallette devra d'ailleurs faire quelques concessions par rapport au refus de toute publicité commerciale au sein de la revue. Dans les années 1900, Paul Léautaud est chargé des démarches publicitaires pour les éditions du Mercure, et dans les années 1910 apparaissent des publicités commerciales pour des chemins de fer, des machines à écrire, des médicaments. Papini refuse cette évolution, mais il refuse aussi l'idée même du réseau des revues, qui implique l'inscription dans une forme de tradition :

\begin{abstract}
Ma il fatto è questo : che il Leonardo è costretto a sparire, oltre che per altre ragioni, perché troppi s'interessavan a noi. Come i nostri lettori ricorderanno più volte ci siamo lamentati di questo superfluo successo. Non solo in Italia, ma in Francia, in Inghilterra, in America, in Germania - perfino a Tien-tsin e al Cairo - c'erano uomini che credevano farci piacere e onore occupandosi delle cose nostre. [...]. Gli intrighi, le rivalità, le bizze, le mutue concessioni, le involontarie ipocrisie, le interessate rivolte, gli umilianti maneggi ; tutto ciò che rende frivola e falsa la vita delle riviste « giovani » ci ha costretti a guardare con occhio meno indulgente le nostre apparenti complicità e solidarietà.
\end{abstract}

[Mais le fait est que Leonardo est contraint à disparaître, outre d'autres raisons, parce que trop de gens s'intéressaient à nous. Comme nos lecteurs s'en souviennent, nous nous sommes lamentés plusieurs fois de ce succès superflu. Pas seulement en Italie, mais en France, en Angleterre, en Amérique, en Allemagne - et même jusqu'à Tien tsin et au Caire - il y avait des hommes qui croyaient nous faire plaisir et honneur en se mêlant de nos affaires. [...] Les intrigues, les rivalités, les caprices, les concessions mutuelles, les hypocrisies involontaires, les révoltes intéressées, les manèges humiliants : tout ce qui rend frivole et fausse la vie des revues «jeunes » nous a obligés à regarder avec un œil moins indulgent nos apparentes complicités et solidarités. $]^{30}$

La Voce, deuxième revue fondée par Papini et Prezzolini en 1908, prend donc une toute autre voie $^{31}$. Ce célèbre périodique hebdomadaire, dont le premier numéro paraît le 20

\footnotetext{
${ }^{28}$ Giovanni Papini et Giuseppe Prezzolini, «La Fine », Leonardo, année V, n³, août 1907, p. 257.

${ }^{29}$ Voir la thèse non publiée de Claire Lesage, Le 'Mercure de France' de 1890 à 1914, thèse de l'École nationale des Chartes, 1985, p. 206. L'auteur cite les souscripteurs pour Une Saison en Enfer de Rimbaud publié par la revue. Y figurent notamment Louis Bathou, président du Conseil des ministres, et Raymond Pointcarré, le président de la République de l'époque (p. 446).

${ }^{30}$ Giovanni Papini et Giuseppe Prezzolini, « La Fine », art. cit..

${ }^{31}$ Walter Binni précise que, si l'on retrouve le duo Papini/Prezzolini, c'est davantage Prezzolini qui donne le ton à La Voce, contrairement à Leonardo, plutôt papinien : « il tono del 'Leonardo' è dato da Papini [...] mentre quello dalla 'Voce' è totalmente prezzoliano. », «Importanza del movimento della 'Voce' », Il Campano, a. XIII, nn.3/4, mai-juin 1935, p. 28, mis en ligne sur le site http://circee.lett.unitn.it, consulté le 5/02/2013. Nous ne revenons pas sur les liens entre La Voce et Les Cahiers de la Quinzaine de Charles Péguy, souvent évoqués par la critique (voir par exemple l'étude de Bruno Somalvico déjà citée, «La Voce et la reformulation de l'identité culturelle italienne »). Hormis peut-être pour les Quaderni della Voce, il s'agit cependant davantage d'une affinité d'esprit et de principe que d'une similitude éditoriale. Pour François Livi, il est d'ailleurs inutile de chercher, comme équivalente française, une revue littéraire («è inutile cercare, come equivalente francese, una rivista letteraria ») ; François Livi, «I francesi nella Voce », dans «La Voce » 1908-2008, a cura di Sandro Gentili, Gabinetto Scientifico Letterario G.P. Vieusseux, Morlacchi Editore, 2010, p. 537. Le critique nuance
} 
décembre 1908, diffère grandement de Leonardo $^{32}$. Son format et son esthétique sont ceux du journal, avec un format in $-4^{\circ}$ sur quatre colonnes, qui deviendra le format traditionnel des revues avant-gardistes italiennes, et avec peu d'illustrations ${ }^{33}$. En janvier 1914, la revue, d'hebdomadaire, devient bimensuelle, et change de format : sous-titrée « Rivista d'idealismo militante », elle revient à un petit format de revue. Comme l'écrit Bruno Somalvico, on peut se demander s'il s'agit d'une revue ou d' « un journal hebdomadaire culturel ». La langue notamment est «vivante et non livresque », avec un «style clair et simple $»^{34}$. Son contenu évoque à la fois les questions culturelles et littéraires et les problèmes de société et de politique, abordés à partir d'un point de vue essentiellement moral ${ }^{35}$. Le ton est donné dès le premier numéro avec un article de Prezzolini sur la politique française, et surtout un article de Papini intitulé «L'Italia risponde». Il y invite les Italiens à se recentrer sur leur culture qui vaut bien celle de l'étranger. Dans le numéro suivant, le 27 décembre 1908, Prezzolini publie un manifeste qui se détache tout à fait des manifestes des «petites revues » françaises : il y insiste sur la réalité politico-sociale de l'Italie, tout autant que sur la dimension culturelle. Il faut cependant noter l'importance de l'expérimentation formelle au sein de la Voce, surtout à partir de 1914, avec la direction de De Robertis et le développement du «frammentismo $»^{36}$. Le succès de La Voce n'est pas sans rappeler celui du Mercure de France. En 1911, La Voce devient une société éditoriale autonome avec un capital et des associés. De plus, la revue crée une maison d'édition dès 1909 : les «Edizioni della Voce». Une seconde collection voit le jour en 1910, «I Quaderni della Voce», en collaboration avec la Casa Editrice Italiana di Firenze des frères Quattrini ${ }^{37}$. Parallèlement à La Voce, en 1911, Papini crée avec Amendola également le rapprochement souvent évoqué avec la $N R F$ : «Se le similitudini tra 'La Nouvelle Revue française' e 'La Voce' non sono trascurabili - la presocché identica data di nascita, la nascita di una casa editrice che affianca l'attività della rivista, la profonda influenza esercitata, al margine del mondo accademico, sulla cultura dell'epoca, con il conseguente affermarsi di 'generi' tipici della rivista, ecc. - la differenze sono vistose. » [Si les similitudes entre La Nouvelle Revue française et La Voce ne sont pas négligeables - la presque identique date de naissance, la naissance d'une maison d'édition qui soutient l'activité de la revue, la profonde influence exercée, aux marges du monde académique, sur la culture de l'époque, avec l'affirmation de «genres » typiques de la revue qui en découle, etc. - les différences sautent aux yeux.] (ibid.).

${ }^{32}$ Il est d'usage de différencier deux Voce : celle qui va de 1908 à 1914, sous la direction de Prezzolini (avec une interruption en 1912-1913, période pendant laquelle La Voce est dirigée par Papini), et celle qui va de 1914 à 1916, appelée la « Voce bianca », sous la direction de Giuseppe De Robertis, essentiellement littéraire.

${ }^{33}$ Les illustrations sont surtout présentes pour les articles de critique d'art, domaine important au sein de $L a$ Voce. Mais contrairement à la revue Leonardo, on ne trouve ni cul-de-lampes, ni vignettes.

${ }^{34}$ Bruno Somalvico, «La Voce et la reformulation de l'identité culturelle italienne », Cahiers Georges Sorel, $\mathrm{n}^{\circ} 5,1987$, p. 131 et 133.

${ }^{35}$ Sur la question de la morale dans La Voce, voir Walter Binni, art. cit.

${ }^{36}$ Sur le mouvement du frammentismo autour de La Voce, voir notamment Arnaldo Bocelli, Il Frammentismo, dans Novecento. I Contemporanei, Milan, Marzorati, 1979 ; Donato Valli, Vita e morte del 'frammento' in Italia, Lecce, Milella, 1980 et Clelia Martignoni, «Sulla letteratura vociana : la riforma dei generi e dello stile », Strumenti critici, VIII, 1993, 2, p. 189-203.

${ }^{37}$ Sur l'expérience éditoriale de La Voce, voir l'analyse très détaillée de Luca Brogioni, « «La Voce », la rivista che volle farsi editore », art. cit.. L'activité éditoriale se poursuivra après l'arrêt de la publication de La Voce en 1916. 
la revue L'Anima, saggi e giudizi, davantage dans l'esprit de Leonardo. Elle prend la forme de fascicules d'une trentaine de pages $(25 \times 18 \mathrm{~cm})$, avec une couverture blanche et une typographie sobre. Revue d'idées encore une fois, à forte dimension philosophique, elle accueille des articles de Giovanni Boine ou Piero Marrucchi. Mais c'est avec Lacerba en 1913 que Papini se dissocie de La Voce, jugée trop modérée. En tant que modèle éditorial, la revue créée par Papini et Soffici, en collaboration avec Italo Tavolato et Aldo Palazzeschi, s'inscrit cependant dans la lignée de La Voce. Éditée par Vallecchi, qui deviendra l'éditeur de l'avant-garde en Italie, la revue paraît de janvier 1913 à mai 1915. Le titre est inspiré d'un poème de Cecco d'Ascoli, L'acerba, dont les premiers vers figurent sur l'en-tête : "Qui non si canta al modo delle rane $»^{38}$. Bimensuelle, puis hebdomadaire à partir de 1915, la revue se présente d'abord sous le format journal, avec seize grandes pages de deux colonnes, puis huit pages la dernière année. Le frontispice, d'abord noir, puis rouge en 1915, est de Soffici, comme l'était déjà celui de La Voce. Lacerba publie des articles de critique, d'art, mais aussi des textes de création. Au fil des livraisons, la revue se rapproche du futurisme et opère une véritable révolution typographique. Elle s'achève par une rubrique «Schiocchezzaio e Spicilegio » (bêtisier et spicilège), qui s'étendra en 1914, avec une importante rubrique «Caffè »: « Notizie echi petegolezzi bottate motti di spirito aneddoti sciochezze polemichette indiscrezioni curiosità sfoghi frasi caratteristiche pulci dell' orecchio ecc $»^{39}$. Le contenu et la rhétorique de Lacerba s'inscrivent davantage dans la lignée de Leonardo, par la tonalité ludique et polémique. Giovanni Papini, après avoir regardé du côté de la France dans sa recherche d'un modèle éditorial de revue d'avant-garde, semble donc avoir trouvé sa propre voie. Néanmoins, des liens persistent avec les revues françaises, et notamment avec le Mercure de France, à travers des écrivains comme Apollinaire, mais aussi avec une figure moins connue des échanges culturels : Remy de Gourmont.

\section{Une figure de passeur : Remy de Gourmont}

La «dissociation des idées » : un exemple de circulation des idées dans le réseau francoitalien des revues

Dès la fin des années 1890, les jeunes revues italiennes se montrent très attentives à l'œuvre de Gourmont. Des articles lui sont consacrés dans Il Marzocco, Flegrea, Leonardo et

\footnotetext{
38 «Ici l'on ne chante pas à la manière des grenouilles ».

39 «Nouvelles échos commérages piques bons mots anecdotes stupidités petites polémiques indiscrétions curiosités défoulements phrases caractéristiques puces à l'oreille etc. »
} 
La Voce, et lui-même collabore à beaucoup d'entre elles. Gourmont est surtout connu par l'intermédiaire du Mercure de France, mais aussi à travers d'autres revues comme La Revue des idées, L'Ermitage ou La Revue indépendante. D'abord considéré comme un représentant du symbolisme, notamment dans le premier Marzocco dirigé par Enrico Corradini, Gourmont est par la suite surtout apprécié en tant que critique et homme de revue. Il est en particulier reconnu pour sa fameuse «dissociation des idées », qui consiste à «imaginer des rapports nouveaux entre les vieilles idées, les vieilles images, ou [de] séparer les vieilles idées, les vieilles images unies par la tradition, [de] les considérer une à une, quitte à les remarier et à ordonner une infinité de couples nouveaux qu'une nouvelle opération désunira encore, jusqu'à la formation toujours équivoque et fragile de nouveaux liens $\gg^{40}$ Or cette dissociation des idées est particulièrement bien illustrée par les «Épilogues », petites chroniques publiées dans le Mercure de France depuis les années 1890 et bien connues dans les réseaux italiens des jeunes revues. Cette théorie, ou méthode, s'adapte parfaitement au support périodique, et surtout à la « rhétorique » de certaines revues françaises de l'époque, qui s'inscrivent de façon ironique, voire ludique, et en tout cas critique, dans l'actualité, et qui prônent le jeu sur les idées et la relativité du monde, en accord avec la philosophie de Schopenhauer ou celle, plus récente, de Bergson. Lecteur assidu du Mercure de France ${ }^{41}$, Papini a aussi eu l'occasion de lire les contributions de l'écrivain français dans Il Marzocco, Flegrea ou La Rassegna internazionale della letteratura e dell'arte contemporanea de Florence ${ }^{42}$. Il admire beaucoup Gourmont, qu'il rencontre à Paris en $1910^{43}$. Soffici quant à lui découvre plus tardivement Gourmont, grâce à Hélène d'Oettingen, dite Yadwiga ${ }^{44}$, lors de son deuxième séjour à Paris,

\footnotetext{
${ }^{40}$ Remy de Gourmont, «Les mots et les idées », Mercure de France, janvier 1900, p. 5.

${ }^{41}$ « e andavo leggendo nel Mercure de France, i suoi saggi, i suoi dialoghi, le sue cronache, dove si respirava sempre un' aria lucida e pungente di alta libertà intellettuale » [et je lisais dans le Mercure de France ses essais, ses dialogues, ses chroniques, dans lesquelles on respirait toujours un air pur et chargé de grande liberté intellectuelle] (Papini, « Remy de Gourmont », Passato remoto, Florence, L'Arco, 1948, repris dans Autoritratti e ritratti, Milan, Mondadori, 1962, p. 933).

42 «In Italia dovrebb'essere conosciuto. Scrisse in riviste italiane : nella Rassegna Internazionale, nel Marzocco e in Lacerba di Firenze, nella Flegrea di Napoli. » [Il devrait être connu en Italie. Il a écrit dans des revues italiennes : dans la Rassegna Internazionale, dans le Marzocco et dans Lacerba de Florence, dans Flegrea de Naples.] (Papini, «Remy de Gourmont», Il Resto del Carlino, 17 octobre 1915, repris dans Stroncature, Valecchi, 1920, $5^{\mathrm{e}}$ édition, p. 189).

${ }^{43}$ Cette visite est racontée par l'écrivain italien dans Passato remoto, Florence, L'Arco, 1948, repris dans Autoritratti e ritratti, Milan, Mondadori, 1962, p. 933-937.

${ }^{44}$ Dans le portrait que Soffici consacre à Gourmont, Soffici souligne le rôle d'Hélène d'Oettingen dans sa rencontre avec l'écrivain français : «Madame era una geniale signora straniera amica dello scrittore francese e mia, alla quale dovevo e di aver avuto il desiderio che ho detto e di ottenere quella risposta cortese. » [Madame était une géniale dame étrangère, amie de l'écrivain et la mienne, à laquelle je devais d'avoir eu le désir dont j'ai parlé et d'obtenir cette courtoise réponse] («Con Remy de Gourmont», Ricordi di vita artistica e letteraria, Florence, Vallecchi, 1942, repris dans Opere VI, Vallecchi, 1965, p. 142).
} 
et grâce à Papini qui l'incite à relire son œuvre ${ }^{45}$. Dans un portrait de l'écrivain français, Soffici revient sur l'importance de la figure de Gourmont pour les jeunes écrivains :

Del resto - a proposito della sua grande simpatia per noi giovanni scrittori italiani - la gioventù in generale trovava costantemente in lui un attento e cordiale estimatore. Nel suo stesso paese, non sorgeva piccola rivista o moto innovatore, ch'egli non l'accogliesse con testimonianze di affettuoso cameratismo. È così che i più audaci gli erano amici [...].

[Du reste - à propos de la grande sympathie qu'il éprouvait pour nous, jeunes écrivains italiens - la jeunesse en général trouvait en lui un estimateur attentif et cordial. Dans son pays même, il n'y avait pas de petite revue ou de mouvement novateur qui ne naquît sans qu'il l'accueillît avec des témoignages d'affectueuse camaraderie. C'est ainsi que les plus audacieux étaient ses amis. $]^{46}$

Mais si les rencontres entre Gourmont, Papini et Soffici ont lieu dans les années 1910, la connaissance de l'œuvre de l'écrivain français est bien antérieure : elle remonte, pour Papini en tout cas, au début des années 1900. Gourmont est régulièrement mentionné dans Leonardo. Outre La Revue des idées, Papini cite La Culture des idées ${ }^{47}$, la $~ «$ dissociation des idées ${ }^{48}$, et Physique de l'amour ${ }^{49}$. On le voit, ce sont surtout les idées de Gourmont et sa méthode dissociative qui intéressent le jeune écrivain italien. L'«idéalisme» des rédacteurs du Leonardo rappelle ainsi par certains aspects les théories gourmontiennes :

\footnotetext{
Nella Vita son pagani e individualisti [...].

Nel Pensiero sono personalisti e idealisti, cioè superiori ad ogni sistema e ad ogni limite, convinti che ogni filosofia non è che un personal modo di vita - negatori di ogni altra esistenza di fuor dal pensiero.

Nell'Arte amano la trasfigurazione ideale della vita e ne combattono le forme inferiori, aspirano alla bellezza come suggestiva figurazione e rivelazione di una vita profonda e serena.
}

[Dans la Vie, ils sont païens et individualistes.

Dans la Pensée, ils sont personnalistes et idéalistes, c'est-à-dire supérieurs à tout système et à toute limite, convaincus que toute philosophie n'est qu'un mode de vie personnel négateurs de toute existence en-dehors de la pensée.

Dans l'Art ils aiment la transfiguration idéale de la vie et en combattent les formes inférieures, ils aspirent à la beauté comme figuration suggestive et révélation d'une vie profonde et sereine. $]^{50}$

Que veut dire Symbolisme? [...] individualisme en littérature, liberté de l'art, abandon des formules enseignées [...]. ${ }^{51}$

La seule réalité, c'est la pensée. ${ }^{52}$

\footnotetext{
${ }^{45}$ Soffici écrit ainsi à Papini en 1910 : «Ho letto anche il Gourmont che conoscevo e ci ho trovato delle belle cose » [J'ai aussi lu Gourmont que je connaissais, et j'y ai trouvé de belles choses] (lettre de Soffici à Papini citée par Mario Richter, op. cit., p. 161).

${ }^{46}$ Ardengo Soffici, « Con Remy de Gourmont », op. cit., p. 147.

${ }^{47}$ Papini, «Chi sono i socialisti ? Socialismo e religione », Leonardo, année 1, n 6,8 mars 1903, p. 1-2.

${ }^{48}$ Gian Falco, « Morte e resurrezione della Filosofia », Leonardo année 1, n 11, 20 décembre 1903, p. 6.

${ }^{49}$ Gian Falco, « Athena e Faust », Leonardo, année III, n² 1, février 1905, p. 14.

${ }^{50}$ «Programma sintetico », Leonardo, année I, 4 janvier 1903, n 1 , p. 1.

${ }^{51}$ Remy de Gourmont, Le Livre des masques, Mercure de France, 1896, p. 8.

${ }^{52}$ Remy de Gourmont, L'Idéalisme, Mercure de France, 1893, p. 13.
} 
Or l'idéalisme des rédacteurs du Leonardo, inspiré notamment de Bergson, est complexe ${ }^{53}$, et c'est sous le nom de «pragmatisme » que Papini et Prezzolini développent leurs idées à partir de la deuxième série de Leonardo, en novembre 1903. Si le pragmatisme doit beaucoup à Bergson, il rejoint également l'idéalisme gourmontien sur plusieurs points : l'importance du particulier et de l'individuel, la problématique du réel et de l'idéal et les potentialités magiques de la pensée (qui rejoignent la théorie du bovarysme développée par Gourmont et Jules de Gaultier ${ }^{54}$ ). Ces liens entre les deux écrivains, à travers le prisme de Schopenhauer et de Bergson, apparaissent dès le premier article que Papini consacre à la philosophie en décembre 1903:

La filosofia farà quel che nessuno fa o vuol fare, ha trovato la sua missione originale : sarà la ricerca e la scoperta del particolare. [...] Gran parte del progresso intellettuale è fatto con dissociazioni d'idee (Remy de Gourmont), e il raffinamento dei sensi consiste nel cogliere quelle differenze che ai sensi rozzi e non esercitati sfuggono.

[La philosophie fera ce que personne ne fait ou ne veut faire, elle a trouvé sa mission propre : ce sera la recherche et la découverte du particulier. [...] Une grande partie du progrès intellectuel est faite avec la dissociation d'idées (Remy de Gourmont), et le raffinement des sens consiste dans la saisie des différences qui échappent aux sens grossiers et non exercés. $]^{55}$

Ci dobbiamo proporre di render concreta la volontà, cioè di rendere reali esternamente $i$ nostri desideri (sogno magico che passa in filosofia). Così l'uomo non solo colla creazione della verità, dell'infinito e della legge si fa Dio (Goethe, Fichte, Feuerbach, Stirner, Comte, Hazard, Maeterlinck), ma anche coll'oggettivazione concreta del suo desiderio, colla creazione della realtà.

[On doit se proposer de rendre la volonté concrète, c'est-à-dire de rendre réels extérieurement nos désirs (songe magique qui passe dans la philosophie). Ainsi l'homme se fait Dieu non seulement avec la création de la vérité, de l'infini et de la loi (Goethe, Fichte, Feuerbach, Stirner, Comte, Hazard, Maeterlinck), mais aussi avec l'objectivation concrète de son désir, avec la création de la réalité. $]^{56}$

\footnotetext{
${ }^{53}$ Papini répond ainsi à un article de Diego Garoglio : «Bisognerà ricordargli che la filosofia della contingenza non è idealista, come basterebbero a provarlo le pagine di Bergson in 'Matière et mémoire' dove combatte ugualmente e idealismo e materialismo. Noi siamo anche idealisti, ma non questo soltanto [...]. » [Il faudra lui rappeler que la philosophie de la contingence n'est pas idéaliste, comme suffiraient à le prouver les pages de Bergson dans Matière et Mémoire, dans lesquelles il combat à la fois l'idéalisme et le matérialisme. Nous sommes aussi idéalistes, mais pas seulement cela [...].] («Palle al Balzo », Leonardo, II, 1, mars 1904, p. 31). Papini commente l'article de Diego Garoglio paru dans Avanti della Domenica le 20 mars 1904 et dans lequel il qualifiait les rédacteurs de la revue d' «ultra-idéalistes ».

${ }^{54}$ Sur le lien entre la dissociation et le bovarysme, voir notre étude sur les chroniques de Remy de Gourmont et d'Alfred Jarry, « De la dissociation à la Pataphysique : lumières d'Alfred Jarry et Remy de Gourmont sur les chooses du temps », L'Étoile-Absinthe, tournées 111-112, 2006, p. 14-19.

${ }^{55}$ Papini, « Morte e resurrezione della filosofia », Leonardo, année I, n 11,20 décembre 1903, p. 1-7, repris dans Sul Pragmatismo (Saggi e ricerche) 1903-1911 [Milan, Libreria Editrice Milanese, 1913], dans Opere I, éd. Luigi Baldacci, Milan, Mondadori, 1977, p. 20-21.

${ }^{56}$ Papini, Sul Pragmatismo, ibid., p. 22-23. Le bovarysme est plus évident encore dans les écrits sur le moi. Voir notamment Un uomo finito, chap. XLVII, « Chi sono » [Florence, Libreria della Voce, 1913] dans Opere I, éd. cit., p. 373 : «Io son rimasto, insomma, l'uomo che non accetta il mondo e in questo mio atteggiamento ostinato consiste l'unità e la concordia delle mie anime opposte. Io non voglio accettare il mondo com'è e perciò tento di rifarlo colla fantasia o di mutarlo colla distruzione. » [«Je suis resté, en somme, l'homme qui n'accepte pas le
} 
Cette volonté est fondée sur un constat : il y a autant de mondes qu'il y a de personnes, et la conclusion de Papini reprend alors le principe énoncé dans Le Livre des masques: "Così non parleremo più di filosofia ma di filosofi, e non faremo la storia delle dottrine ma la storia dei dottrinari $»^{57}$. Cette conclusion est significative du refus de système, et donc d'une certaine vision de la philosophie. Là encore, l'auteur italien rejoint Gourmont dans la conception d'une vérité relative, qui s'applique tout aussi bien à la philosophie qu'à la science ${ }^{58}$. On comprend mieux alors tout l'intérêt de Papini et Prezzolini pour La Revue des idées, qui rejette selon eux la dimension religieuse de la science, c'est-à-dire l'idée d'une science comme vérité absolue. S'opposant à tout système philosophique, Papini prône le jeu, qui acquiert ses lettres de noblesse à la suite du rire de Bergson et de la dissociation des idées de Gourmont :

[...] il giuoco, inteso in tutta la sua profonda ricchezza, non è stato ancora elevato agli onori di principio ispiratore dell'intera vita. Dirò di più : il giuoco è disdegnato e sospetto come qualcosa tra il fanciullesco e il delinquente, e non gode troppo le simpatie degli autori dei libri di testo delle giovini generazioni.

[...] Tutta l'arte della vita, ch'è per me la scienza suprema, consiste nello scegliere i più bei giuochi, i passatempi superiori.

[[...] le jeu, conçu dans toute sa profonde richesse, n'a pas encore été élevé à l'honneur de principe inspirateur de toute la vie. Je dirai même plus : le jeu est dédaigné et soupçonné d'être quelque chose entre l'enfantin et le délinquant, il ne jouit guère des sympathies des auteurs de livres des jeunes générations.

[...] Tout l'art de la vie, qui est pour moi la science suprême, consiste à choisir les plus beaux jeux, les passe-temps supérieurs. $]^{59}$

Papini s'intéresse donc particulièrement aux «Épilogues » de Gourmont, qui constituent une application de ces principes et de cette esthétique dans le support périodique. Les Épilogues semblent d'ailleurs avoir attiré l'attention d'autres écrivains italiens. Beaucoup des comptes rendus des petites revues italiennes du début des années 1900 portent sur ces chroniques, qui apparaissent comme un exemple abouti de dissociation des idées, mais aussi de fragmentation :

monde et en cette attitude obstinée réside l'unité et l'harmonie de mes âmes opposées. Je ne veux pas accepter le monde tel qu'il est, c'est pourquoi j'essaie d'en reconstruire un avec l'imagination ou de le changer avec la destruction. »] On reconnaît également la méthode de la dissociation des idées qui agit sur le monde par une destruction théorique.

${ }^{57}$ Papini, Sul Pragmatismo, ibid., p. 24 : «Ainsi nous ne parlerons plus de philosophie mais de philosophes, et nous ne ferons pas l'histoire des doctrines, mais celle des doctrinaires. »

${ }^{58}$ Voir le développement sur la «non-vérité » de Laura Schram Pighi dans Bergson e il bergsonismo, op. cit., p. 147-166. Une fois encore, Bergson apparaît comme la source principale de Papini et Prezzolini, mais Gourmont vient vraisembablement compléter la lecture du philosophe. L'absence de vérité absolue est l'un des thèmes favoris de Gourmont, notamment dans les « Épilogues ».

${ }^{59}$ Gian Falco, «Piccoli e grandi giuochi », Leonardo, année I, n 4,8 février 1903, p. 2-3. 
Ma il segreto della sua forza liberatrice sta proprio in quella sua delicata virtuosità di scomporre i pensieri apparentemente semplici, di separare le coppie supposte indivisibili, di ristabilire i raccordi e le prospettive fra l'idee credute eterogenee e lontane, di frugare nelle spezzature della verità, di svestire cortesemente le più solenni verità per metter sotto gli occhi spaventati l'ossa ignude della contraddittoria verità.

[Mais le secret de sa force libératrice réside justement dans cette délicate virtuosité de décomposer les pensées apparemment simples, de séparer les couples supposés indivisibles, de rétablir les raccords et les perspectives entre des idées que l'on croit hétérogènes et éloignées, de fouiller dans les poubelles des préjudices pour retrouver les éclats de la vérité, de déshabiller courtoisement les plus solennelles vérités pour mettre sous les yeux effrayés l'ossature nue de la contradictoire vérité. ${ }^{60}$

Les rencontres entre Gourmont, Papini et Soffici en 1910 et 1911 concrétisent donc un lien qui était présent, bien que souterrain, dès la fondation de Leonardo. On comprend mieux alors l'importance que revêt le soutien de Gourmont à la revue Lacerba. Lors de la création de la revue en 1913, Soffici écrit à Papini :

Ieri sono stato da Remy de Gourmont. Uomo eccellente e fortificante. Mi ha detto che la Voce non l'interessava più affatto, che è contento che prenda un'altra strada: mi ha promesso di collaborare alla nostra rivista che faremo.

[Hier je suis allé chez Remy de Gourmont. Homme excellent et qui nous fortifie. Il m'a dit que la Voce ne l'intéressait plus du tout, qu'il est content qu'elle prenne une autre voie : il m'a promis de collaborer à la revue que nous ferons. $]^{61}$

De fait, Gourmont collabore au troisième numéro avec des Pas sur le sable inédits. Ces courtes pensées s'accordent parfaitement avec l'esprit de la revue, voire avec la rhétorique qu'elle met en place ${ }^{62}$ : symptôme de la liberté qui caractérise la pensée de Gourmont, sous forme de contradiction, d'ironie et de paradoxe, elles s'inscrivent dans la continuité des «Épilogues » publiés dans le Mercure de France :

Un homme d'esprit n'est pas un homme intelligent ; c'est souvent le contraire. [...]

Soyez humains : si vous avez un fils qui ne sait pas distinguer les couleurs, faites en plutôt un critique d'art qu'un mécanicien de chemin de fer. [...]

Loin de vivifier le style, l'épithète le fige. Les êtres et les choses n'ont pas qu'une apparence, ils en ont mille et dans le même instant. [...]

Le monde: des forces en folie. Partout la difformité partout le hasard, partout l'équivoque..$^{3}$

\footnotetext{
${ }^{60}$ Papini, « Remy de Gourmont », dans Stroncature, éd. cit., p. 192.

${ }^{61}$ Lettre de Soffici à Papini, citée par Mario Richter dans La Formazione francese di Ardengo Soffici, éd. cit., p. 197.

${ }^{62}$ Walter Binni parle ainsi de la «rhétorique » de la revue Leonardo : «Soprattutto il 'Leonardo' tendeva a un europeizzamento italiano in funzione di una rivolta ad ogni passato ed a ogni retorica : era anche questa, a suo modo, una retorica, ma almeno si trattava di una retorica giovane contro una retorica vecchia, senza ragioni di vita. » [Surtout, le Leonardo tendait à une européanisation italienne à partir d'une révolte envers tout passé et toute rhétorique : c'était aussi, d'une certaine manière, une rhétorique, mais il s'agissait au moins d'une jeune rhétorique contre une vieille rhétorique, sans raison de vivre] ( Importanza del movimento della 'Voce' », art. cit., p. 1).

${ }^{63}$ «Des pas sur le sable », Lacerba, I, n³, $1^{\text {er }}$ février 1913.
} 
Prezzolini, quant à lui très critique envers Gourmont, souligne lui aussi l'importance de la dissociation des idées à l'époque :

\begin{abstract}
L'idea più originale che abbia avuto è quella da lui detta « dissociazione delle idee ». Egli prende due idee comunemente associate nella vita, e dimostra che razionalmente sono disgiunte. Egli adopra quello strumento di separazione che è l'intelligenza e capisce benissimo che l'intelligenza è soltanto dissociativa. [...] In Italia cotesto modo di fare alla Gourmont è da parecchi anni di moda.

[L'idée la plus originale qu'il ait eue est celle qu'il appelle « dissociation des idées ». Il prend deux idées communément associées dans la vie, et il démontre qu'elles sont rationnellement disjointes. Il adopte cet instrument de séparation qu'est l'intelligence et il comprend parfaitement que l'intelligence est seulement dissociative. [...] En Italie, cette manière de faire est à la mode depuis quelques années. $]^{64}$
\end{abstract}

Il insiste d'ailleurs à deux reprises, a posteriori, sur le rôle de Gourmont dans la revue Lacerba, d'abord dans une lettre à Soffici : «[Lacerba] la quale non ebbe origini nazionali, ma anarchiche, internazionaliste, menimpiste, amorali, come si vede benissimo dal $1^{\circ}$ anno di essa, inspirato a quel prototipo di decadente che era Remy de Gourmont ${ }^{65}$; puis dans un article de La Coltura italiana sur les revues qui critique l'esthétique de Papini à l'époque : «Papini, che iniziò la collaborazione con piccoli paradossi ghignanti alla Rémy de Gourmont $»^{66}$. La présentation du premier numéro de la revue, «Introibo », formule en effet quelques principes proches de l'esthétique de l'écrivain français :

2. Un pensiero che non può esser detto in poche parole non merita d'esser detto.

3. Chi non riconosce agli uomini d'ingegno, $[\ldots]$ agli artisti il pieno diritto di contraddirsi da un giorno all'altro non è degno di guardarli. [...]

6. Libertà. Non chediamo altro ; chiediamo soltanto la condizione elementare perché l'io spirituale possa vivere. $[\ldots]$

11. Noi amiamo la verità fino al paradosso (incluso) $[\ldots]$.

[2. Une pensée qui ne peut être dite en peu de mots ne mérite pas d'être dite.

3. Celui qui ne reconnaît pas aux hommes d'esprits, [...] aux artistes le plein droit de se contredire d'un jour à l'autre n'est pas digne de les regarder.

6. Liberté. Nous ne demandons rien d'autre; nous demandons seulement la condition essentielle pour que le moi spirituel puisse vivre.

11. Nous aimons la vérité jusqu'au paradoxe (inclus). $]^{67}$

\footnotetext{
${ }^{64}$ Prezzolini, «L'uomo libero : Remy de Gourmont», dans Uomini 22 e città 3, Florence, Vallecchi, 1920, p. 185 et 187. Prezzolini oppose à l'intelligence la pensée unifiante qui manquerait à l'écrivain français (p. 186).

${ }^{65}$ «Laquelle n'eut pas d'origines nationales, mais anarchistes, internationalistes, menimpistes, amorales, comme cela se voit très bien dans la première année de celle-ci, inspirée de ce prototype décadent qu'était Remy de Gourmont. » (Lettre 562 de Prezzolini à Soffici, New York, 10 janvier 1939, Carteggio Prezzolini-Soffici II. 1920-1964, éd. de M.E. Raffi et Mario Richter, Rome, Edizioni di Storia e Letteratura, 1982, p. 113).

${ }^{66}$ «Papini, qui commença sa collaboration avec des petites paradoxes sarcastiques à la Remy de Gourmont. » Prezzolini, « Le riviste », dans La Coltura italiana, Firenze, Soc. An. Editrice « La Voce », 1923, p. 154.

${ }^{67}$ «Introibo », Lacerba, I, n 1,1 janvier 1913.
} 
La dissociation des idées, fondée sur la liberté absolue, la contradiction et le paradoxe, est ainsi reprise par les écrivains italiens au sein de leurs propres revues, tant sur le principe que sur la forme.

\title{
La circulation des formes : le fragment critique du principe philosophique à l'éclatement formel
}

Dans la préface à Euvres de Soffici, Prezzolini revient sur les origines du frammentismo :

\begin{abstract}
[...] quella poetica e quel gusto che furono propri, in Italia, degli scrittori posteriori al D'Annunzio, raccolti idealmente intorno alla Voce...

Al centro di questa poetica è il concetto di poesia come brevità, immediatezza autobiografica, folgorazione lirica dei sensi, fuori d'ogni disegno e struttura : cioè l'idea che la poesia non possa essere che a frammenti e, come tale, inconciliabile con la narrativa, la drammatica e, in genere, con ogni forma di letteratura «costruita $»$ e « oggettiva ».

[[...] cette poétique et ce goût qui furent propres, en Italie, aux écrivains postérieurs à D'Annunzio, rassemblés idéalement autour de La Voce... Au centre de cette poétique se trouve le concept de poésie comme brièveté, immédiateté autobiographique, fulguration lyrique des sens, en-dehors de tout dessin et structure : c'est-à-dire l'idée que la poésie ne puisse se réaliser que dans les fragments, et, comme telle, soit inconciliable avec la narration, l'art dramatique, et, en général, avec toute forme de littérature «construite » et «objective». $]^{68}$
\end{abstract}

Or à côté des œuvres narratives souvent citées, comme Il mio Carso de Slataper, Ragazzo de Jahier, Arlecchino de Soffici ou Frammenti lirici de Rebora, il faut accorder une place aux écrits critiques publiés dans les revues, évidemment dans La Voce, mais aussi dans Leonardo et Lacerba. Dépassant l'opposition entre prose et poésie, le fragment brouille également la frontière entre littérature, philosophie et critique. Il découle ainsi d'une vision du monde et d'une théorie du langage et de la littérature héritée de la France et de l'Allemagne. La revue Leonardo est exemplaire du lien établi entre la philosophie et la littérature française et allemande, et plus précisément entre Bergson et le Romantisme allemand ${ }^{69}$. Papini et Prezzolini consacrent de nombreux articles et comptes rendus à la littérature romantique, représentative à leurs yeux de l'anti-positivisme tout autant que Bergson. Prezzolini rapproche

\footnotetext{
${ }^{68}$ Définition attribuée par Prezzolini à Arnaldo Bocelli, dans la préface à Opere de Soffici, éd. cit., p. XXIII. Cette définition se trouve dans Novecento. I contemporanei, Milan, Marzorati, 1979, p. 1241. Sur le frammentismo, voir également Enrico Falqui, Ragguaglio sulla prosa d'arte, Florence, 1954, et « Sorrentino e il frammentismo », dans Novecento letterario, série 5, Florence, 1957, p. 277-281; Donato Valli, Vita e morte del «frammento » in Italia, Lecce, Milella, 1980 ; Pieter de Meijer, La Prosa narrativa moderna, in Letteratura italiana. Le forme del testo. II. La prosa, sous la direction d'Alberto Asor Rosa, Torino, Einaudi, 1984 ; Oreste Macri, «Poetica del frammentismo e genere del frammento », dans La Vita della parola. Studi su Ungaretti e poeti coevi, sous la direction d'Anna Dolfi, Roma, Bulzoni, 1998.

${ }^{69}$ Voir à ce sujet Anne-Rachel Hermetet, « Romantisme et bergsonisme dans la revue florentine Leonardo», art. cit.
} 
ainsi Novalis de Bergson et des «décadents » français, expression que l'on retrouvera sous sa plume à propos de Gourmont :

\begin{abstract}
Nei primi anni del secolo XIX fu in Germania un decadente ; e la sua teoria estetica si avvicina singolarmente a quella dei decadenti francesi, come fu espressa dal Bergson ; con questo ha in comune il senso di un io più profondo e nascosto, origine di ciò che è creazione.

[Dans les premières années du XIX ${ }^{\mathrm{e}}$ siècle il fut en Allemagne un décadent; et sa théorie esthétique se rapprochait singulièrement de celle des décadents français, telle qu'elle fut formulée par Bergson; avec lui, il a en commun le sens d'un moi plus profond et caché, origine de ce qui est création. $]^{70}$
\end{abstract}

L'écrivain italien insiste plus particulièrement sur la forme fragmentée de son écriture, qu'il rapproche de l'esthétique de Leonardo: «Fece opera frammentaria, come noi, per suggerire pensieri come nei suoi versi ebbe in mira la musica, per suggerire ritmi $»^{71}$. Un lien se tisse entre l'anti-positivisme, les penseurs allemands et français :

Chi ha ravvicinato [...] il Bergson ai romantici tedeschi, s'è un po'troppo soffermato su somiglianze di formule : mentre c'era da notare, e non secondaria, questa parentela di spirito, che gli uni come l'altro, eran stati di quei gassificatori del filosofismo loro contemporaneo, ed avevan segnato un rimpuerilimento del pensiero sotto moltissimi aspetti eccellente [...]. Non si parla di Kant, per molti aspetti chiusura dell'Aufklärung più che apertura del romanticismo, ma dei filosofi del sentimento dell'intuizione e del linguaggio : di questi filosofi a frammenti e frammentatori di filosofie.

[Qui a rapproché [...] Bergson des romantiques allemands s'est un peu trop arrêté sur les ressemblances de formules: alors qu'il fallait noter, et cela n'est pas secondaire, cette parenté d'esprit, que les uns comme l'autre avaient été de ceux qui avaient gazifié le philosophisme contemporain, et avaient marqué une rénovation de la pensée, excellente par bien des aspects. [...] On ne parle pas de Kant, qui clôt par bien des aspects L'Aufklärung plus qu'il n'ouvre le romantisme, mais des philosophes du sentiment, de l'intuition et du langage : de ces philosophes à fragments et fragmentateurs de philosophies. $]^{72}$

Par cette parenté d'esprit plus que de contenu, Gourmont a tout à fait sa place dans la lignée des penseurs «fragmentaires et fragmenteurs», principalement par sa théorie de la dissociation des idées. Le premier article de Prezzolini sur Bergson dans le quatrième numéro du Leonardo se réfère implicitement à la dissociation gourmontienne, preuve que la lecture de Bergson et celle de Gourmont sont étroitement liées. S'attardant sur « la misère de la logique», Giuliano il Sofista commente l'Essai sur les données immédiates de la

\footnotetext{
${ }^{70}$ Giuliano il Sofista, «E. Spenlé, Novalis : essai sur l'idéalisme romantique en Allemagne », Leonardo, année II, n² 2 , juin 1904, p. 35. Le «décadent» désigne Novalis. Prezzolini a traduit ses Fragments : Frammenti, Milan, Libreria Editrice Lombardia, 1905.

${ }^{71}$ «Il fit une œuvre fragmentaire, comme nous, pour suggérer des pensées, de même que dans ses vers il visa la musique, pour suggérer des rythmes. » («E. Spenlé, Novalis : essai sur l'idéalisme romantique en Allemagne», ibid., p. 34).

${ }^{72}$ «Il Bergson », La Voce, II, 4, 6 janvier 1910, p. 239. Nous soulignons.
} 
conscience $^{73}$. Reprenant «les paroles mêmes » de Bergson dans son ouvrage ${ }^{74}$, il montre que la logique ne peut rendre compte du fonctionnement complexe de la vie intérieure : «il divenire continuo, la novità assoluta di ogni istante della coscienza escludono da essa il principio di identità, tipo del perfetto intelligibile logico $»^{75}$. Le jeu sur les idées est dans l'esprit de Prezzolini en parfait accord avec les principes bergsoniens. Se servant de la logique tout en la détruisant, il répond à l'aspiration du nouveau « sophiste » italien :

\begin{abstract}
Servirsi della logica per diminuirla dell'impero che ha sulle nostre menti, negarla senza rendercela aliena, di padrona imperiosa farla schiava obbediente e di necessità del pensiero pura possibilità di ragionamento, spezzarne i muri, escir fuori delle sue rotaie per aprirsi una via verso conoscenze più vaste e più libere, innalzarsi tanto da scorgerla sotto di noi immiserita dalla distanza, nebbiosa per la bassezza, e contornata come un piccolo regno da vastissimi imperi, è cosa che mi tenta.
\end{abstract}

[Se servir de la logique pour diminuer l'empire qu'elle a sur nos esprits, la nier sans se la rendre hostile, de patronne impérieuse la rendre esclave obéissante, et de nécessité de la pensée la rendre pure possibilité de raisonnement, en casser les murs, sortir de ses ornières pour s'ouvrir une voie vers des connaissances plus vastes et plus libres, s'élever au point de l'apercevoir en-dessous de nous, rendue misérable par la distance, embrumée par la bassesse, et encerclée comme un petit règne de très vastes empires, c'est quelque chose qui me tente. $]^{76}$

En concluant son article par ces mots : «Il pensiero è un gioco », « la pensée est un jeu », Prezzolini s'associe à Papini pour esquisser une ligne directrice de la revue, posée implicitement dans la lignée de Gourmont ${ }^{77}$.

Le premier numéro de Leonardo débute par un article de Gianfalco, «L'ideale imperialista ». Papini y oppose la conception d'un impérialisme intellectuel à celui d'un impérialisme guerrier. L'idée et le ton même de l'article rappellent « Le Joujou patriotisme » de Gourmont ${ }^{78}$. À la domination par les armes et la guerre, Papini répond par la domination de l'intelligence. C'est bien la même dissociation qu'opérait Gourmont entre le patriotisme territorial et le patriotisme intellectuel. Mais la forme de l'article se rapproche davantage des écrits postérieurs d'un Gourmont qui associe la méthode de la dissociation d'idées à la fragmentation, avec une nette tendance à la juxtaposition. «L'ideale imperialista » est divisé en deux parties numérotées, qui, sous une argumentation apparente, voilent un goût pour la

\footnotetext{
${ }^{73}$ Giuliano il Sofista [Prezzolini], «La miseria dei logici », Leonardo, année I, n4, 8 février 1903, p. 5-7.

${ }^{74}$ Ibid., p. 6.

75 «le devenir continu, la nouveauté absolue de chaque instant de la conscience excluent pour elle le principe d'identité, type du parfait intelligible logique. » (Ibid., p. 6).

${ }^{76}$ Ibid., p. 5.

${ }^{77}$ Giuliano il Sofista, « La miseria dei logici. II », Leonardo, année I, n 6,8 mars 1903, p. 8.

${ }^{78}$ Remy de Gourmont, «Le Joujou patriotisme », Mercure de France, avril 1891. Ce virulent pamphlet contre le nationalisme et l'esprit de revanche a fait scandale en France et a entraîné le renvoi de l'écrivain de son poste à la Bibliothèque Nationale.
} 
fragmentation, avec de nombreux retours à la ligne et des liens logiques qui tendent à s'effacer au profit de l'énumération :

\begin{abstract}
Voi intendete cioè la potenza in senso soldatesco e saccheggiatore all'uso di Cesare e di Napoleone, che pensavano soprattutto alla schiavitù e distruzione degli uomini e alla conquista della preda.

Voi disdegnate o dimenticate cioè la forza spirituale, la vittoria intellettuale o sentimentale. I vostri eroi e i vostri modelli sono dei soldati, dei condottieri con poche idee, che agiscono più che non pensano.

[C'est-à-dire que vous entendez la puissance au sens soldatesque et pilleur, comme l'ont utilisée César et Napoléon qui pensaient surtout à l'esclavage, à la destruction des hommes et à la conquête de la proie.

Vous dédaignez ou oubliez la force spirituelle, la victoire intellectuelle ou sentimentale.

Vos héros et vos modèles sont des soldats, des meneurs avec peu d'idées, qui agissent plus qu'ils ne pensent. $]^{79}$
\end{abstract}

Cette tendance est plus nette encore dans « Piccoli e grandi giuochi », publié dans Leonardo le 8 février 1903. Papini y refuse explicitement l'organisation traditionnelle de l'article, jouant à la fois sur le rejet de la philosophie comme discipline universitaire et sur le détournement des règles rhétoriques :

S'io fossi uno di quei filosofi dabbene che camminano colle seste e colle squadre e non movon passo senza mostravene il come e il perchè e vi squadernano a ogni pagina la loro brava definizione, io dovrei cominciare col definire il giuoco.

Ma io non tengo ad essere un filosofo galantuomo e da vero masnadiero intellettuale mi permetto di fare a meno di complimenti. Chi ha il gusto di quelle piccole trappole logiche che si chiamano definizioni, se le può fabbricare in famiglia e son certo che gli piaceranno molto più delle mie.

[Si j'étais l'un de ces philosophes comme il faut qui ne font pas un pas sans en montrer le comment et le pourquoi et qui vous étalent à chaque page leur brave définition, je devrais commencer par définir le jeu. Mais je ne tiens pas à être un galant philosophe et, en vrai brigand intellectuel, je me permets de m'en passer. Qui a le goût de ces petits pièges logiques que l'on appelle définitions peut se les fabriquer chez lui, et je suis sûr qu'elles lui plairont beaucoup plus que les miennes. $]^{80}$

L'organisation formelle de l'article reflète le contenu thématique du texte : le jeu prôné par Papini s'oppose aux grandes phrases et aux grands mots, il est du côté de la légèreté et de la désorganisation. Cette forme de jeu semble très proche de la dissociation des idées gourmontienne qui apparait comme un jeu avec les idées, fondé sur une forte dimension ironique et ludique et sur une volonté de désorganisation, voire de destruction. Papini précise en effet sa prédilection pour les «giuochi d'idee » : «Fra gli innumerevoli indici di libri che non farò mai ne conservo uno sulle costruzioni metafisiche, uno sui sofismi, un altro sui metodi migliori per 'épater le bourgeois' e via dicendo. ${ }^{81}$

\footnotetext{
${ }^{79}$ Gianfalco, «L'ideale imperialista », Leonardo, année I, n¹, 4 janvier 1903, p. 2.

${ }^{80}$ Gianfalco, « Piccoli e grandi giuochi », Leonardo, année I, n 4, 8 février 1903, p. 1-2.

81 «Parmi les innombrables listes de livres que je ne ferai jamais, j'en conserve un sur les constructions métaphysiques, un sur les sophismes, un autre sur les méthodes pour 'épater le bourgeois', et ainsi de suite. »
} 
De même, Prezzolini recourt dès l'époque de Leonardo à une esthétique proche de la note. En mars 1904, il publie dans la revue «Manipoli. Elogio delle parole », petit texte qui décline sur un mode ludique diverses idées à partir de la notion de «parole». L'article tend à la parataxe, accentuée par la répétition du groupe nominale «le parole », et par le retour à la ligne :

Le parole servono a inverniciare di virtù i nostri difetti, a stuccare le coscienze incrinate, a vestire di bei muscoli le persone troppo ossute.

Le parole sono come gli abiti : false ed utili. Sono cortigiani e adulatori, che innalzano i nostri fatti alle stelle, cantano la nostra persona, ingrandiscono i nostri pensamenti. Sono un fôro di avvocati ben pagati, un areopago di giudizi ben disposti, una schiera di militi devoti.

[Les paroles servent à maquiller de vertu nos défauts, à plâtrer les consciences lézardées, à vêtir de beaux muscles les personnes trop osseuses.

Les paroles sont comme les vêtements : fausses et utiles. Elles sont des courtisanes et des adulatrices, qui élèvent nos actes jusqu'aux étoiles, chantent notre personne, agrandissent nos pensées. Elles sont un forum d'avocats bien payés, un aéropage de juges bien disposés, un groupe de soldats dévots. $]^{82}$

Il s'agit déjà d'un jeu sur les idées, d'une décomposition à partir de la notion de « paroles », associée à diverses images.

Cette tendance à la fragmentation, esquissée dans les pages de Leonardo, s'accentue avec l'expérience de La Voce. Dans les années 1910, époque du rapprochement entre Gourmont, Papini et Soffici, la revue publie des articles de plus en plus fragmentés, des aphorismes et des pensées de Schlegel ou Hebbel ${ }^{83}$. En 1914, Prezzolini inaugure dans La Voce une rubrique dont le titre met en évidence l'héritage gourmontien: «Scomposizioni d'idee». La décomposition est proche de la dissociation, comme le montre l'exercice du 13 mars 1914 sur les notions d' « errore » et de « sbaglio », « erreur » et « faute » :

\begin{abstract}
Siamo in un momento in cui molti li confondono. Sarà dunque bene vedere per che cosa si distinguono ; ed anche che cosa significhi la confusione che se ne fa. Essa stessa è un errore o uno sbaglio?

[Nous sommes dans un moment où beaucoup les confondent. Il sera donc bon de voir ce qui les distingue ; et également ce que signifie la confusion que l'on fait. Est-elle elle-même une erreur ou une faute ? $]^{84}$
\end{abstract}

Le fonctionnement de la rubrique souligne le lien entre la méthode dissociative et la forme fragmentée. Après avoir présenté le sujet choisi, Prezzolini développe une série de réflexions plus ou moins longues, nettement séparées entre elles par des carrés noirs. La brièveté peut

\footnotetext{
(Ibid., p. 3).

${ }^{82}$ Giuliano il Sofista, «Elogio delle parole », Leonardo, année II, n ${ }^{\circ}$, mars 1904, p. 19.

${ }^{83}$ Voir par exemple «Pensieri di Federigo Schlegel», La Voce, II, 2, 23 décembre 1909, p. 230-231, et « Pensieri di F. Hebbel », La Voce, II, 14, 17 mars 1910, p. 287.

${ }^{84}$ Prezzolini, « Scomposizioni d'idee. Errore e sbaglio », La Voce, VI, 5, 13 mars 1914, p. 40.
} 
aller jusqu'à l'aphorisme : «Chiarire la propria espressione è innalzarsi eticamente ${ }^{85}$. Cette esthétique, à laquelle tendent les écrivains dès les années 1890, trouve donc un terrain particulièrement fertile dans les pages des revues. C'est là qu'elle se développe, qu'elle se systématise et se théorise, culminant dans les pages de Lacerba.

Dans une lettre à Rodolphe Darzens, Ephraïm Mikhaïl décrit la stratégie éditoriale d'une « revue qui ne serait pas faite comme les autres », la future Pléiade : «Voilà à peu près ce que nous voudrions faire. Le principe est une revue à très petit nombre d'exemplaires (200 ex.) et de rédacteurs (six ou sept). ${ }^{86}$ Cela semble correspondre tout à fait à la notion de « petite revue ». Cependant, l'expression rend mal compte d'une réalité mouvante. Il est difficile d'établir un modèle éditorial figé pour des revues comme le Mercure de France, La Plume, L'Ermitage ou La Revue des idées. Il est néanmoins possible de discerner quelques traits dominants de ces revues très lues par les jeunes écrivains florentins. Le premier concerne l'oscillation entre la revue et le journal, tant au niveau matériel qu'au niveau du contenu. Le deuxième relève de l'hybridité entre la modernité et le caractère ancien de ces périodiques, par la dimension bibliophilique et par l'importance de l'image. La troisième touche au projet de rénovation culturelle plus ou moins ambitieux : les périodiques de l'époque veulent faire entendre la voix des jeunes et aspirent souvent à révolutionner l'art et la culture de l'époque. Enfin, la revue offre une autonomie éditoriale recherchée par de jeunes auteurs qui veulent s'imposer sur la scène littéraire sans pour autant faire des concessions commerciales. Toutes ces caractéristiques se retrouvent dans Leonardo, et il est évident que les «petites revues » parisiennes, comme les appelle Papini lui-même, ont joué un rôle dans l'élaboration des stratégies éditoriales de l'avant-garde florentine. On retrouve également dans les revues de Papini l'esprit provocateur et ludique des revues parisiennes de la fin du XIX ${ }^{\mathrm{e}}$, et, comme nous avons tenté de le montrer, une rhétorique particulière. C'est dans ce sens que la figure de Gourmont émerge du réseau franco-italien des revues. En 1914, l'écrivain français est cité par Papini dans «Ce que nous devons à la France » pour sa «manière de décomposer, de renverser et de dénuder les idées communes ${ }^{87}$. Car l'esthétique de l'avant-garde florentine,

\footnotetext{
${ }_{85}$ «Clarifier sa propre expression, c'est s'élever éthiquement.» («Scomposizioni d'idee. Dell'oscurità dei giovani », La Voce, VI, 1, 2 janvier 1914, p. 19).

${ }^{86}$ Ephraïm Mikhaël, lettre à Rodolphe Darzens du 30 janvier 1886 à propos de La Pléiade, dans Ephraïm Mikhaïl, Euvres Complètes. Aux origines du Symbolisme, second volume, Lausanne, Éditions L'Age d'Homme, 2001, p. 363.

87 « una maniera di scomporre, rovesciare e spogliare le idee comuni » («Ciò che dobbiamo alla Francia», Lacerba, II, $\mathrm{n}^{\circ} 17,1^{\mathrm{er}}$ septembre 1914).
} 
comme celle de Gourmont, est étroitement liée à l'écriture en revue, non seulement parce qu'elle constitue le mode de publication privilégié d'une esthétique moderne et expérimentale, mais aussi parce que la poétique périodique s'accorde avec la conception artistique de ces auteurs. La fragmentation en constitue un exemple significatif, répondant à la fois à une vision du monde éclatée par la modernité et le refus des règles, et aux conditions d'écriture propre au périodique.

Alexia Kalantzis, article paru dans la Revue de littérature comparée, n³51, 2014/3, p. 285-309. 